\title{
Characterization and Recognition of Point-Halfspace and Related Orders
}

\author{
(Preliminary Version)
}

\author{
Paul J. Tanenbaum ${ }^{1 \star}$, Michael T. Goodrich ${ }^{2 \star \star}$, and Edward R. Scheinerman ${ }^{3}$ \\ 1 Department of Computer Science, The Johns Hopkins University, \\ Baltimore, Maryland 21218-2694 \\ pjtocs.jhu.edu \\ and U.S. Army Research Laboratory, \\ Aberdeen Proving Ground, Maryland 21005-5068 \\ pjtoarl.mil \\ 2 Department of Computer Science, The Johns Hopkins University, \\ Baltimore, Maryland 21218-2694 \\ goodrichecs. jhu . edu \\ ${ }^{3}$ Department of Mathematical Sciences, The Johns Hopkins University, \\ Baltimore, Maryland 21218-2689 \\ ersecs.jhu. edu
}

\begin{abstract}
We characterize four classes of geometric membership and containment orders-structurally and in terms of forbidden subposetsand present linear- or near linear-time recognition algorithms for each class. We also show that recognizing point-halfspace orders in $\mathbb{R}^{d}$ is NPhard for $d \geq 2$.
\end{abstract}

\section{Introduction}

The study of hierarchical relations among geometrical objects is an area rich in mathematical structure. Such relations often provide efficient means for representing and manipulating data. One example is the point-halfspace graphs introduced by Scheinerman, Trenk, and Ullman [26]. These are graphs in which the vertices are points in $\mathbb{I}^{d}$ and the edges are halfspaces. The same article introduces point-halfspace orders, those partially ordered sets (posets) representable in terms of points and halfspaces ordered by membership, and asks for a characterization of them. We address that question and several closely related ones here. The posets we consider are those represented by sets of points and various convex subsets of $\mathbb{R}^{d}$.

\subsection{Related Work}

Characterizing properties in terms of obstructions, and in particular forbidden restrictions, is well known in discrete mathematics. The most famous such characterization is Kuratowski's Theorem: The planar graphs are exactly those containing no subdivisions of $K_{5}$ or $K_{3,3}$ (see for instance Bondy and Murty [2]).

* This research is supported in part by NSF grant CCR-9300079.

* This research is supported by NSF under grants IRI-9116843 and CCR-9300079. 
Others include the observation that the bipartite graphs are those containing no odd cycles and Fishburn's characterization of interval orders [15] as those containing no $2+2$. Some of the many classes of containment orders that have been studied are the sphere orders (balls in $\mathbb{R}^{d}$ as studied, for instance, by Brightwell and Winkler [4]) and the angle orders (see for instance Fishburn and Trotter [16]).

Recognition algorithms have also been developed for many classes of graphs and posets. Hopcroft and Tarjan [22] presented the first $O(n+m)$-time algorithm to recognize planar graphs (see also [9], [13], [3], [8], and [5]). Besides a planarity test, Booth and Lueker [3] also give a linear-time algorithm to recognize interval graphs, which are also treated in $[23,24,25,28]$. Other important recognition problems include visibility graphs $[6,7,14,19]$, comparability graphs $[20,29,24]$, and series-parallel graphs $[1,12,32]$. A final recognition algorithm-one we will use in this paper-is that of Spinrad and Valdes [30], an $O\left(n^{2}\right)$-time algorithm for the posets of dimension less than or equal to 2. For more information on testing graph properties, please see the survey by Di Battista et al. [10].

On the other hand, the recognition problem is difficult for several important classes. For example, determining whether a graph has a Hamilton cycle is NPcomplete (see Garey and Johnson [17]) and Garg and Tamassia [18] have recently shown that while graph planarity can be answered in linear time, the problem of determining whether a poset has a planar Hasse diagram is NP-complete.

\subsection{Our Results}

In this paper we address membership and containment orders in $\mathbb{R}^{d}$ for points and halfspaces and for points and convex sets. In describing algorithms that manipulate posets $(X, \leq)$ we refer to the size of the input in terms of the parameters $n$ and $m$, where $n=|X|$ is the size of the ground set and $m=|\leq|$ is the number of pairs $x \leq y$ in the partial order. We adopt the same convention for graphs, letting $n$ be the number of vertices and $m$ the number of edges.

After some preliminary definitions and conventions in section 2, we ascertain in sections 3-6 the forbidden restrictions for each of the four classes for $d=1$ and describe their structures. For the two point-ray classes we provide $O(n+m)$ time recognition algorithms. In the case of point-interval membership orders we give a reduction to interval-graph recognition to obtain an $O\left(n^{2}\right)$-time algorithm based on the results in Booth and Lueker [3] and Simon [28] and for point-interval containment orders we prove the sufficiency of the $O\left(n^{2}\right)$-time algorithm in [30]

Given the characterizations of these four classes of orders, it is natural to ask how the classes are related. In section 7 we present the containment relations among them. In section 8 we consider higher dimensions, proving that the recognition of point-halfspace orders is NP-hard in $\mathbb{R}^{d}$ for $d>1$.

\section{Preliminary Definitions and Conventions}

Let $P=(X, \leq)$ be a poset-a set $X$ together with a relation $\leq$ on $X$ that is reflexive, antisymmetric, and transitive. Alternatively, $P$ may be viewed as the 
transitive closure of an acyclic digraph with vertex set $X$ and arc set $\leq$. Any $x \in X$ is minimal (resp. maximal) in $P$ provided there is no $y \in X$ such that $y<x$ (resp. $x<y$ ). The set of all minimals (resp. maximals) of $P$ is denoted $\min (P)($ resp. $\max (P))$. A chain in $P$ is a set of elements $x_{1}<\cdots<x_{t}$ of $X$. The height of $P$ is the size of a maximum chain.

For any relation $R$ on a set $X$ and any $x \in X$ we denote by $X_{R}(x)$ the set of all $y \in X$ such that $y R x$. The downset of an element $x$ in $P$ (the set of all $y \in X$ such that $y \leq x)$ is thus denoted by $X_{<}(x)$. The strict downset is the set $X_{<}(x)=X_{\leq}(x) \backslash\{x\}$. The upset $X_{\geq}(x)$ and strict upset $X_{>}(x)$ are defined analogously. If neither $x \leq y$ nor $y \leq x$ for $x, y \in X$, then we call $x$ and $y$ incomparable in $P$ and write $x \| y$. The width of $P$ is the size of a largest set of pairwise incomparable elements.

A linear order is a partial order in which no pair is incomparable. Dushnik and Miller [11] defined the dimension of a poset $P=(X, \leq)$ as the size of a smallest set of linear orders on $X$ whose intersection is $\leq$. Equivalently, the dimension is the smallest $d$ such that there is an order-preserving embedding of $P$ in $\mathbb{R}^{d}$ with

$$
\left(x_{1}, \ldots, x_{d}\right) \leq\left(y_{1}, \ldots, y_{d}\right) \text { if and only if }\left(x_{1} \leq y_{1}\right) \wedge \cdots \wedge\left(x_{d} \leq y_{d}\right)
$$

Trotter [31] provides an excellent introduction to the theory of poset dimension.

A poset $P^{\prime}=\left(X^{\prime}, \leq^{\prime}\right)$ is a restriction of the poset $P=(X, \leq)$ provided $X^{\prime} \subseteq X$ and for all $x, y \in X^{\prime}, x \leq^{\prime} y$ whenever $x \leq y . P^{\prime}$ may be obtained from $P$ by deleting elements from $X$ and exactly those comparabilities in $\leq$ that involve the deleted elements. $P^{\prime}$ is also called an induced subposet of $P$. A bipartite poset is an ordered triple $(B, T, \leq)$ where $(B \cup T, \leq)$ is a poset in which the only comparabilities are of the form $b<t$ and $x=x$, for $b \in B, t \in T$ and $x \in B \cup T$.

A poset $P=(X, \leq)$ is a $\Sigma$-containment order for some class $\Sigma$ of sets if there is a function $f: X \rightarrow \Sigma$ mapping elements of the ground set to sets in the class so that for $x, y \in X$ we have $x \leq y$ precisely when $f(x) \subseteq f(y)$. Similarly, $P$ is a $\mathcal{B}-\mathcal{T}$-membership order for a set $\mathcal{B}$ and a collection $\mathcal{T}$ of subsets of $\mathcal{B}$ if there is a function $g: X \rightarrow \mathcal{B} \cup \mathcal{T}$ for which $x<y$ precisely when $g(x) \in \mathcal{B}$, $g(y) \in \mathcal{T}$, and $g(x) \in g(y)$. Although we choose to use the language of order theory, a $\mathcal{B}-\mathcal{T}$-membership order may also be thought of as a hypergraph with vertex set $\mathcal{B}$ and edge set $\mathcal{T}$. The functions $f$ and $g$ are called $\Sigma$-containment and $\mathcal{B}-\mathcal{T}$-membership representations, respectively. Note that every membership order has height 2, but that containment orders can have large heights.

Posets are presented in the figures in terms of their Hasse diagrams: digraph drawings in which arcs all point upward, so arrowheads are omitted, and any arc inferable from transitivity is also omitted.

In this paper we are concerned with two issues: (1) characterizations of containment and membership orders in terms of structural properties and forbidden restrictions and (2) efficient recognition algorithms that can be derived from these characterizations. We announce the characterizations here: their proofs will appear in a forthcoming publication. 


\section{Point-Ray Membership Orders}

We consider first the class of posets isomorphic to some $(X, €)$, where $X$ is a set of points and rays in $\mathbb{R}^{1}$ and $\subseteq$ is the reflexive closure of the is-a-member-of relation $\in$ on $X$. Such a poset is called a point-ray membership order.

\subsection{Forbidden-Restriction Characterization}

We have the following characterization of the point-ray membership orders in terms of forbidden restrictions.

Theorem 1. A bipartite poset is a point-ray membership order if and only if it contains none of the posets in figure 1 as a restriction.

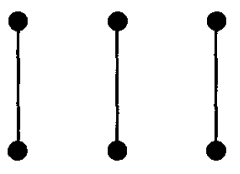

(a) $2+2+2$

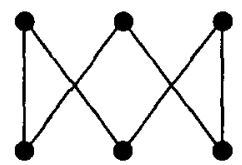

(b) $\mathbf{S}_{3}$

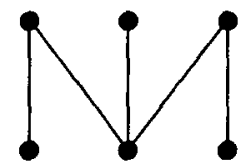

(c) $\mathrm{MI}$

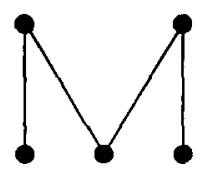

(d) M.

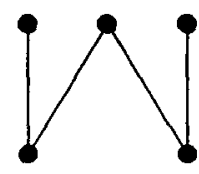

(e) W.

Fig. 1. Forbidden restrictions for point-ray membership orders

\subsection{Structural Characterization}

A poset $(X, \leq)$ is a proper arch provided it has a decomposition $X=C_{1} \cup C_{2}$ into distinct nonempty chains such that $C_{1} \cap C_{2}=\{\hat{x}\}=\max \left(C_{1}\right)=\max \left(C_{2}\right)$ for some $\hat{x} \in X$ and $x \| y$ for all $x \in C_{1} \backslash\{\hat{x}\}$ and $y \in C_{2} \backslash\{\hat{x}\}$. A poset $P$ is an archand is said to be arched-provided it is a restriction of a proper arch. Figure 2(a) illustrates a proper arch and figures 2(b)-(d) illustrate improper arches.

Theorem 2. A poset is an arch if and only if it has width at most 2 and it contains neither of the posets in figure 9 as a restriction.

The top containment poset of a height- 2 poset $P$ is the set $\max (P)$ ordered by containment of strict downsets. 


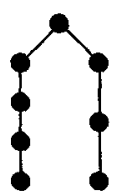

(a)

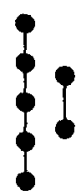

(b)

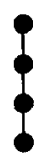

(c)

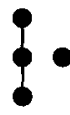

(d)

Fig. 2. Some example arches

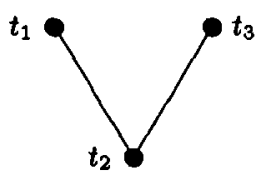

(a) V

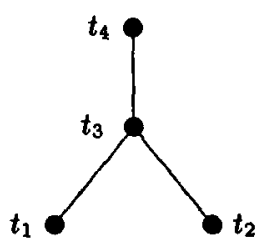

(b) Claw

Fig. 3. Forbidden restrictions for arches

Theorem 3. $A$ bipartite poset $P$ with top containment poset $P^{\prime}$ is a point-ray membership order if and only if $P$ does not contain $M_{0}$ as a restriction; $P^{\prime}$ is an arch; and, if $P^{\prime}$ is proper, then $P$ has no isolated minimals.

Theorems 1 and 3 provide two elegant characterizations of the point-ray membership orders, as well as some insights into their structure. We next show how to exploit these insights.

\subsection{Recognition Algorithm}

Our algorithm is based on theorem 3 . We determine whether a bipartite poset $P=(B, T, \leq)$ is a point-ray membership order and, if so, we construct a representation that also yields an optimal Hasse diagram. The bipartite poset $P$ is input as lists $\left(b_{1}, \ldots, b_{r}\right)$ and $\left(t_{1}, \ldots, t_{s}\right)$ representing $B=\min (P)$ and $T=$ $\max (P)$, respectively, and the adjacency lists $T_{>}\left(b_{i}\right)$ and $B_{<}\left(t_{j}\right)$ for $1 \leq i \leq r$ and $1 \leq j \leq s$.

The requirement that $P$ be a bipartite poset is only necessary in applications where the distinction between points and rays is critical for any element comparable to no other element-an isolate. In an application that allows isolates to be partitioned arbitrarily between points and rays, any poset may be preprocessed in $O(n+m)$ time to test for height 2 and produce a bipartition. In this case, all isolates should be assumed to be maximals.

In its initialization step our algorithm bucket-sorts the maximals $t_{j}$ into buckets $\beta_{k}$ for $0 \leq k \leq r$ containing all the maximals with strict downsets of size $k$. It then checks each of the three conditions of theorem 3 .

To ensure that the top containment poset $P^{\prime}$ of $P$ is an arch we check the buckets by decreasing $k$, trying to build two chains $C_{1}$ and $C_{2}$ of downsets 
ordered by inclusion. Let $c_{1}^{-}$and $c_{2}^{-}$be the minimals of $C_{1}$ and $C_{2}$, respectively. Let $c_{1}^{+}$and $c_{2}^{+}$be the maximals of $C_{1}$ and $C_{2}$, respectively, unless $\max \left(C_{1}\right)=$ $\max \left(C_{2}\right)$, in which case, call the maximal of both chains $c^{+}$and let $c_{1}^{+}$and $c_{2}^{+}$be the maximals of $C_{1}-c^{+}$and $C_{2}-c^{+}$, respectively. It then follows from theorem 2 that $P^{\prime}$ is an arch if and only if $c_{1}^{-} \nsubseteq c_{2}^{+}$and $c_{2}^{-} \nsubseteq c_{1}^{+}$. Next, if $P^{\prime}$ is a proper arch, which is to say if $\max \left(C_{1}\right)=\max \left(C_{2}\right)$, we check for an isolated minimal.

The last step in the algorithm is to test whether $P$ contains $M_{0}$, which we can do in linear time thanks to one more structural result. Define a function $\alpha_{1}$ on $B$ such that $\alpha_{1}(b)=k$ whenever $b$ appears in $C_{1} \cap \beta_{k}$ but not in $C_{1} \cap \beta_{j}$ for any $j<k$. Define $\alpha_{2}$ analogously for $C_{2}$. Then we have

Proposition 4. Let $P$ be a poset of which the top containment poset $P^{\prime}$ is an arch with chain decomposition $C_{1} \cup C_{2}$. Then $P$ contains $M_{0}$ if and only if there exist minimals $x$ and $y$ of $P$ such that $\alpha_{1}(x)<\alpha_{1}(y)$ and $\alpha_{2}(x)<\alpha_{2}(y)$.

The remaining task of building a point-ray membership representation $f$ of $P$ is straightforward. An ascent of $C_{1}$ and a descent of $C_{2}$ maps the bottoms onto $\{1, \ldots, r\}$. Then the tops in $C_{1}$ may be mapped to leftward rays and those in $C_{2}$ to rightward rays. This also provides a blueprint for a Hasse diagram: $B$ may be sorted onto the line $y=0$ by increasing $f$ and $T$ onto $y=1$ by increasing height in $C_{1}$ and then decreasing height in $C_{2}$. Furthermore, it is not difficult to show that of the Hasse diagrams of $P$ drawn with $B$ on one line, $T$ on another, and straight-line edges, those that follow this blueprint have the smallest possible number of edge crossings.

\section{Point-Ray Containment Orders}

For the next class of posets we augment the "point $\epsilon$ ray" relations of the previous section with "ray $\subset$ ray" relations. More precisely, we consider the point-ray containment orders, those posets isomorphic to $(X, \subseteq)$ for some $X \subseteq$ $\{\{x\} \mid x \in \mathbb{R}\} \cup\{$ rays along $\mathbb{R}\}$.

\subsection{Forbidden-Restriction Characterization}

We enumerate the forbidden restrictions in figure 4 using a modified Hassediagram convention: the solid circles represent minimals and the open circles nonminimals. Thus each open circle must be understood to have at least one element below it.

Theorem 5. A poset is a point-ray containment order if and only if it contains none of the posets in figure 4 as a restriction.

\subsection{Recognition Algorithm}

This algorithm is similar to the one for point-ray membership orders in section 3.3. We determine whether a poset $P=(X, \leq)$ is a point-ray containment order and, if so, we construct a representation. 


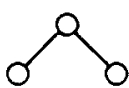

(a)

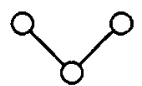

(b)
000

(c)

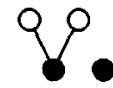

(d)

[NOTE: Minimals are shown solid and nonminimals open.]

Fig. 4. Forbidden restrictions for point-ray containment orders

$P$ is input as a list $\left(x_{1}, \ldots, x_{n}\right)$ representing $X$ and the adjacency lists $X_{<}\left(x_{i}\right)$ and $X_{>}\left(x_{i}\right)$ for $1 \leq i \leq n$. We initialize the input by labeling each $x_{i}$ as minimal or nonminimal. We bucket-sort the nonminimals into buckets $\beta_{k}$ for $0 \leq k<n$ containing all the nonminimals $x_{j}$ for which $\left|X_{<}\left(x_{j}\right)\right|=k$. We then determine whether $P$ is a point-ray containment order in two phases. In the first phase we check for the obstructions in figure 4(a)-(c) and in the second we check for the one in figure $4 \mathrm{~d}$. This can all be done in $O(n+m)$ time.

\section{Point-Interval Membership Orders}

The next class of posets we consider is again constrained to height 2 . We replace the rays of the point-ray membership orders with intervals to obtain the pointinterval membership orders.

\subsection{Structural Characterization}

Let $P=(X, \leq)$ be a height-2 poset with $\min (P)=\left\{b_{1}, \ldots, b_{n}\right\}$. The topintersection graph of $P$ is the graph with vertex set $\max (P)$ in which any two elements of $\max (P)$ are adjacent if and only if they are above a common minimal. If $P$ is bipartite, we define the augmentation of $P$ as the poset obtained by adding for each $b_{i}$ a new top above $b_{i}$ and nothing else. The augmented top-intersection graph of $P$ is the top-intersection graph of the augmentation of $P$. As we show in the following theorem, this graph provides a nice structural characterization of point-interval membership orders.

Theorem 6. A bipartite poset is a point-interval membership order if and only if its augmented top-intersection graph is an interval graph.

\subsection{Forbidden-Restriction Characterization}

The forbidden restrictions for point-interval membership orders consist of two explicit posets and three infinite families. In the Hasse diagrams in figure 5 we have highlighted certain vertices and edges to clarify patterns in the obstructions. This highlighting is only to clarify similarities among the posets. All three infinite families are elaborations on the same fence of size $n$-the zigzagging poset indicated by the solid circles and edges. 
Theorem 7. A height-2 poset is a point-interval membership order if and only if it contains none of the posets in figure 5 as a restriction.

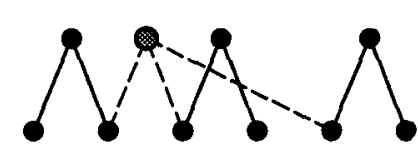

F

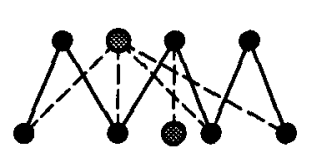

H

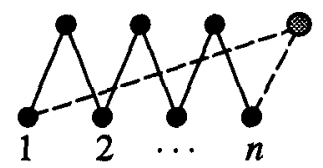

Crown $_{n}, n \geq 3$
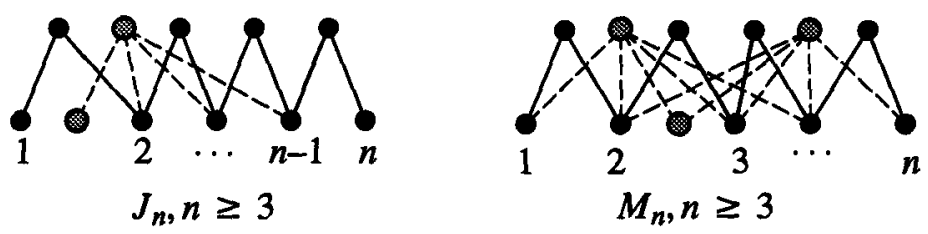

Fig. 5. Forbidden restrictions for point-interval membership orders

\subsection{Recognition Algorithm}

It is not clear how one might exploit the forbidden-restriction characterization in theorem 7 to obtain a recognition algorithm for point-interval membership orders. But a straightforward algorithm follows from theorem 6 . One simply builds the augmented top-intersection graph, which requires $\Theta\left(n^{2}\right)$ time, and then checks whether it is an interval graph. The second step can be done quickly using algorithms published in [3] or [28].

\section{Point-Interval Containment Orders}

The final class of real-line orders we consider is the point-interval containment orders.

Theorem 8. The point-interval containment orders are exactly the interval containment orders, which is to say the posets of dimension at most 2. Moreover, every poset of dimension at most 2 has a point-interval containment representation that maps all its minimals to points.

\subsection{Recognition Algorithm}

As an immediate result of theorem 8 , the point-interval containment orders can be recognized in $O\left(n^{2}\right)$ time using the algorithm in [30]. 


\section{Containments Among the Classes}

In theorems 9 through 11 we detail the containment relationships among the four classes of posets that we have considered so far. These containments are summarized in figure 6 . The posets included in the figure are certificates that each of the illustrated regions is nonempty. The dashed circle in the figure indicates that, as stated in theorem 10 , the containment is true only of those point-interval containment orders that have height 2 or less.

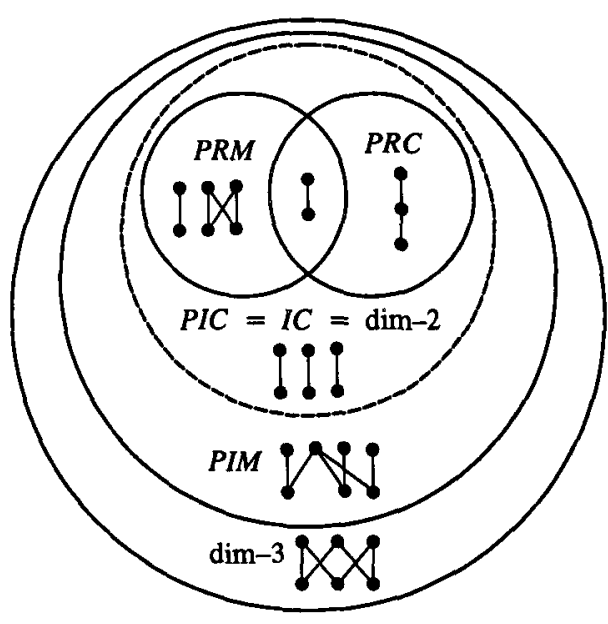

Fig. 6. Containments among the classes

Theorem 9. Point-ray membership orders and point-ray containment orders are all point-interval containment orders.

Theorem 10. The height-2 dimension-2 posets form a proper subset of the pointinterval membership orders.

Theorem 11. Every point-interval membership order has dimension 9 or less.

\section{Point-Halfspace Orders in 2 and More Dimensions}

In this section we show that the recognition of point-halfspace orders in $\mathbb{R}^{d}$ is NP-hard for all $d \geq 2$. Thus it is unlikely that any simple characterization exists for these orders. We begin with the 2-dimensional case and then extend that result to $d>2$. 
The point-halfplane result is obtained by a reduction from a variant of a problem called Pseudoline Stretchability (see Shor [27]). A pseudoline is (the Cartesian graph of) a continuous function $\psi: \mathbb{R} \rightarrow \mathbb{R}$. A set of pseudolines is a collection provided any pair of elements that intersect do so at exactly one point, where they cross. A collection of pseudolines is simple provided no three of them are concurrent and every pair intersect. It is stretchable if its arrangement - the partition of $\mathbb{R}^{2}$ induced by the pseudolines, together with all the incidences-is isomorphic to an arrangement of (straight) lines.

We now formally state the recognition problem for the 2-dimensional case:

POINT-HALFPLANE ORDER

INSTANCE: A bipartite poset $P=(B, T, \leq)$.

QUESTION: Is there an injection $f$ from $B \cup T$ to $\mathbb{R}^{2} \cup\{$ halfplanes\} with $f(B) \subset$ $\mathbb{R}^{2}$ and $f(T) \subset$ \{halfplanes\} such that for all $b \in B$ and $t \in T, f(b) \in f(t)$ if and only if $b<t$ ?

\section{Theorem 12. Point-HalfPlane ORDER is NP-hard.}

Proof. Both Pseudoline Stretchability and the variant in which the input collection of pseudolines is required to be simple are NP-hard [27]. We demonstrate a polynomial reduction from this variant. The arrangement is input as an allowable sequence, as defined by Goodman and Pollack [21]. We assign to every cell a unique bit string that encodes its position relative to each pseudoline and build a bipartite poset $P$ such that $\min (P)$ is the cell labels and $\max (P)$ the pseudolines, and $c<\psi$ precisely when cell $c$ is above $\psi$. This construction, which is illustrated in figure 7, can be done in polynomial time and $P$ is a point-halfplane order if and only if the collection is stretchable.

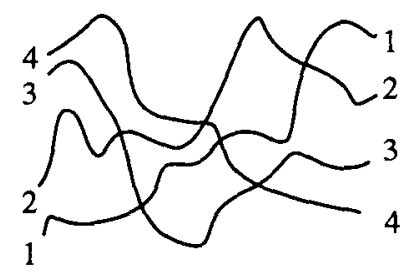

a) Arrangement of Pseudolines

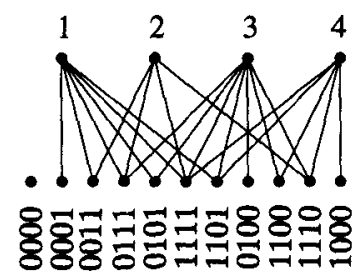

c) Bipartite Poset $P$

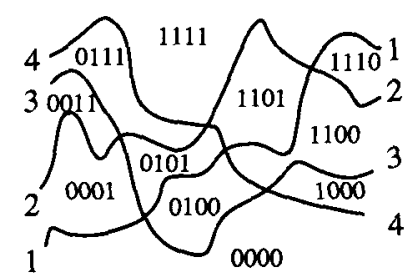

b) Cell Labels

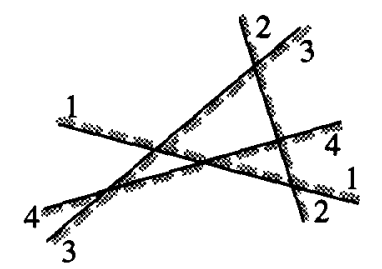

d) Isomorphic Line Arrangement

Fig. 7. Reduction from Pseudoling Stretchabitity 
Not surprisingly, the problem remains hard in higher dimensions. We make this assertion precise with

Theorem 13. Recognition of point-halfspace orders in $\mathbb{R}^{d}$ is $N P$-hard for $d \geq 2$.

Proof. We prove the case $d=3$ with a reduction from PoINT-HalfPlane ORDER. The higher dimensions are handled inductively. Given a bipartite poset $P=(B, T, \leq)$, produce the poset $P^{\prime}=\left(B \cup B^{\star}, T, \leq^{\prime}\right)$ by adding a twin for every minimal of $P$, and then produce $P^{\prime \prime}=\left(B \cup B^{\star}, T \cup\left\{t_{0}\right\}, \leq^{\prime \prime}\right)$ such that $\left(B \cup B^{\star}\right)_{\leq \prime \prime}\left(t_{0}\right)=B^{\star}$. Clearly, $P^{\prime \prime}$ can be constructed in polynomial time and is a point-halfspace order in $\mathbb{R}^{3}$ if and only if $P$ is a point-halfplane order.

\section{References}

1. P. Bertolazzi, R. F. Cohen, G. Di Battista, R. Tamassia, and I. G. Tollis. How to draw a series-parallel digraph. In Proc. 3rd Scand. Workshop Algorithm Theory, volume 621 of Lecture Notes in Computer Science, pages 272-283. Springer-Verlag, 1992.

2. John Adrian Bondy and U. S. R. Murty. Graph Theory with Applications. NorthHolland, New York, 1976.

3. Kellogg S. Booth and George S. Lueker. Testing for the consecutive ones property, interval graphs, and graph planarity using $P Q$-tree algorithms. J. Comput. System Sci., 13:335-379, 1976.

4. Graham Brightwell and Peter Winkler. Sphere orders. Order, 6:235-240, 1989.

5. N. Chiba, T. Nishizeki, S. Abe, and T. Ozawa. A linear algorithm for embedding planar graphs using $P Q$-trees. J. Comput. System Sci., 30(1):54-76, 1985.

6. P. A. Colley. Recognizing visibility graphs of uni-monotone polygons. In Proc. 4th Canad. Conf. Comput. Geom., pages 29-34, 1992.

7. J. Czyzowicz, I. Rival, and J. Urrutia. Galleries, light matchings and visibility graphs. In Proc. 1st Workshop Algorithms Data Struct., volume 382 of Lecture Notes in Computer Science, pages 316-324. Springer-Verlag, 1989.

8. H. de Fraysseix and P. Rosenstiehl. A depth-first-search characterization of planarity. Ann. Discrete Math., 13:75-80, 1982.

9. N. Deo. Note on Hopcroft and Tarjan's planarity algorithm. J. ACM, 23:74-75, 1976.

10. G. Di Battista, P. Eades, R. Tamassia, and I. G. Tollis. Algorithms for drawing graphs: an annotated bibliography. Preprint, Dept. Comput. Sci., Brown Univ., Providence, RI, November 1993. To appear in Comput. Geom. Theory A ppl. Preliminary version available via anonymous ftp from vilma.cs.brown. edu, gdbiblio.tex.Z and gdbiblio.ps. $Z$ in /pub/papers/compgeo.

11. B. Dushnik and E. W. Miller. Partially ordered sets. Amer. J. Math., 63:600-610, 1941.

12. David Eppstein. Parallel recognition of series-parallel graphs. Inform. and Comput., 98(1):41-55, May 1992.

13. S. Even and R. E. Tarjan. Computing an st-numbering. Theoret. Comput. Sci., 2:339-344, 1976.

14. H. Everett and D. G. Corneil. Recognizing visibility graphs of spiral polygons. $J$. Algorithms, 11:1-26, 1990.

15. Peter C. Fishburn. Interval Orders and Interval Graphs: A Study of Partially Ordered Sets. John Wiley, New York, 1985. 
16. Peter C. Fishburn and William Thomas Trotter. Angle orders. Order, 1:333-343, 1985.

17. Michael R. Garey and David S. Johnson. Computers and Intractability: $A$ Guide to the Theory of NP-Completeness. Freeman, New York, 1979.

18. Ashim Garg and Roberto Tamassia. On the computational complexity of upward and rectilinear planarity testing. Technical Report CS-94-10, Brown University, 1994.

19. S. K. Ghosh. On recognizing and characterizing visibility graphs of simple polygons. In Proc. 1st Scand. Workshop Algorithm Theory, volume 318 of Lecture Notes in Computer Science, pages 96-104. Springer-Verlag, 1988.

20. P. C. Gilmore and A. J. Hoffman. A characterization of comparability graphs and of interval graphs. Canad. J. Math., 16:539-548, 1964.

21. Jacob E. Goodman and Richard Pollack. Semispaces of configurations, cell complexes of arrangements. J. Combin. Theory Ser. A, 37:257-293, 1984.

22. J. Hopcroft and R. E. Tarjan. Efficient planarity testing. J. Assoc. Comput. Mach., 21:549-568, 1974.

23. Korte and Möhring. An incremental linear-time algorithm for recognizing interval graphs. SIAM J. Comput., 18, 1989.

24. D. Kozen, U. Vazirani, and V. Vazirani. NC algorithms for comparability graphs, interval graphs, and testing for unique perfect matching. Foundations of Software Technology and Theoretical Computer Science, 1985.

25. Ramalingam and Rangan. New sequential and parallel algorithms for interval graph recognition. Inform. Process. Lett., 34, 1990.

26. Edward R. Scheinerman, Ann N. Trenk, and Daniel Ullman. On point-halfspace graphs. J. Graph Theory, to appear.

27. Peter W. Shor. Stretchability of pseudolines is NP-hard. In P. Gritzman and B. Sturmfels, editors, Applied Geometry and Discrete Mathematics: The Victor Klee Festschrift, volume 4 of DIMACS Series in Discrete Mathematics and Theoretical Computer Science, pages 531-554. Amer. Math. Soc., Providence, R.I., 1991.

28. Klaus Simon. A new simple linear algorithm to recognize interval graphs. In Computational Geometry-Methods, Algorithms and Applications, International Workshop on Computational Geometry $C G$ '91, number 553 in Lecture Notes in Computer Science, pages 289-308. Springer-Verlag, Berlin, 1991.

29. Jeremy Spinrad. On comparability and permutation graphs. SIAM J. Comput, 1985.

30. Jeremy Spinrad and Jacobo Valdes. Recognition and isomorphism of two dimensional partial orders. In Automata, Languages and Programming, 10th Colloquium, number 154 in Lecture Notes in Computer Science, pages 676-686. Springer-Verlag, Berlin, 1983.

31. William Thomas Trotter. Combinatorics and Partially Ordered Sets: Dimension Theory. Johns Hopkins Series in Mathematical Sciences. Johns Hopkins Univ. Press, Baltimore, 1992.

32. J. Valdes, R. Tarjan, and E. Lawler. The recognition of series parallel digraphs. In Proc. 11th ACM Symposium on Theory of Computing (STOC), pages 1-12, 1979. 